\title{
Development of Thinking Mode and Decision-making System for Clinical Nursing Health Assessment Based on Internet + and Evaluation of Its Application Effect
}

\author{
Chang Sun $^{1}$, Zhang-bo Xiao, ${ }^{2, *}$ Xin-jin Cui ${ }^{1}$, Li-li Jiang ${ }^{1}$, Liu Chao ${ }^{1}$ \\ ${ }^{1}$ Qiqihar Institute of Engineering, Qiqihar, 161000, Heilongjiang, China; \\ ${ }^{2}$ The Second Affiliated Hospital of Qiqihar Medical University, Qiqihar, 161000, Heilongjiang, China. \\ *corresponding author: xzbmd@qmu.edu.cn
}

Keywords: Internet+, clinical nursing, health assessment, system development.

\begin{abstract}
With the development of society and scientific progress, people pay more and more attention to health and medical services, and nursing becomes more and more important in the medical process. Active and effective nursing can not only improve the quality of nursing, but also help patients to reply. High-quality nursing can not be carried out without rigorous analysis. From the perspective of social development at this stage, the Internet has penetrated into every corner of our life, and it also plays an important role in clinical nursing health. Now our country's hospital nursing, generally based on the Internet to reform, ushered in a new Internet innovative thinking, and has been confirmed in practice, so that the nursing effect of our country has a qualitative leap. The innovative thinking of clinical care on the Internet + effectively improves the level of clinical care, improves the quality of hospital services, and builds a more trustworthy image for the hospital. This article will analyze and present the development and use effect evaluation of the thinking mode and decision system of clinical nursing health assessment based on Internet + , and provide a reference for Internet+ clinical nursing.
\end{abstract}

\section{Introduction}

Health assessment is an important part of nursing procedure. Only after comprehensive and accurate health assessment can correct nursing diagnosis be put forward to ensure the follow-up overall nursing service quality. The virtual health assessment system based on Internet+ is directed by the competency of nursing students, on-the-job nurses and public health assessment knowledge needs. It uses computer virtual reality technology to construct specific clinical nursing practice situation and simulates the medical clinical nursing practice process through computer human-computer interaction technology. It can provide powerful decision support and health warning for users. Health assessment is a science to study the basic theory, basic skills and clinical thinking methods of diagnosing individual, family or community response to existing or potential health problems or life processes. It is also a basic skill that nurses must possess to provide high-quality nursing service. Clinical health assessment mainly includes the collection of patients' basic data, assessment of symptoms and psychosocial aspects, nursing physical examination, access to relevant data, analysis and induction of evaluation results and corresponding nursing diagnosis. At the same time, it also provides basis for the formulation and implementation of follow-up nursing plan and measures.

\section{Thinking Mode of Clinical Nursing Health Assessment Based on Internet+}

Health assessment is the most basic of all nursing subjects. It links nursing and clinical nursing, transforms book learning into a bridge of practice, plays a very important role in real practice, and has a far-reaching impression on every medical staff in clinical practice. Scientific thinking mode of health assessment and decision-making ability of nursing practice, as the core skills of clinical 
nurses, need to contact patients for a long time, in multiple ways, in order to continuously improve. Behind the thinking of health assessment is the accumulation of constantly updated medical knowledge and clinical nursing experience. With the development of information technology, the way to obtain information is more convenient, and the improvement of public awareness of health is bound to put forward higher requirements for nursing staff's ability level and professional quality. Only relying on clinical practice to improve nursing staff's ability can't cope with the development of the times. A brand-new learning method is urgently needed to reduce the risk of clinical nursing practice, improve the learning quality and promote patient safety.

Internet is a huge library, where you can find all the information you want and find many resources for health assessment. Now we can make full use of Internet technology for health assessment, take clinical symptoms as the main branch, combine Internet technology with medical technology, and conduct theoretical study, analysis and feedback through the Internet.

To sum up, we can pass the clinical nursing health assessment in Internet+. In this way, we can quickly grasp the patient's information, which is more open and shared, and more convenient.

\section{Development of Clinical Nursing Health Evaluation Decision System in Internet+}

\subsection{Competency based model}

Traditional learning is teacher-centered, but because the knowledge taught by different teachers cannot be unified, it is difficult to ensure that all learners can receive the same education. On the other hand, due to the different needs of different learners, centralized teaching may make some learners have to face some learning projects that have nothing to do with them, which leads to inefficiency and waste of time.

Competency-based learning is a discipline-oriented method, which first determines the health problems to be solved, then determines the abilities that nursing students should have in nursing work, then adjusts the curriculum to enable students to have these abilities, and finally evaluates the successes and mistakes. Students can freely choose courses and learning methods in learning, as long as they finally acquire the competence required in different periods. Competency based learning reflects highly individual learning rather than traditional single curriculum.

At the same time, through the simulation study of virtual patients, it can effectively reduce the danger in the nursing process and indirectly avoid nurse-patient disputes. This is very important for training inexperienced nurses.

\subsection{The learning mode of combination of virtual and reality}

Every ability used by users in the virtual diagnosis and treatment system can only be acquired after passing the individual ability examination in the nursing skill center, and then the ability can be used in the virtual diagnosis and treatment system for more comprehensive and advanced learning activities. In other words, the training of thinking logic is carried out through the cheap virtual platform based on internet plus, and then more realistic training is carried out through expensive simulation equipment, which further reduces the risk of real clinical nursing practice.

\subsection{Internet+ based medical database}

Attract different users to participate in the collection and collation of training data based on internet plus platform, and gradually build medical knowledge base by means of public labeling and correction. The difference between wiki technology and traditional wiki technology is that it not only records the knowledge points of nursing, but also extracts the knowledge of diagnosis and treatment process by recording the learning operation process. And it is structured, which is convenient for monitoring the later health assessment process, and reusing the process knowledge of health assessment and nursing practice for similar diseases. 


\subsection{Broad target group}

Through the virtual health assessment system, the geographical restrictions are diluted and the audience of formal education is expanded.

Nursing students: Applying virtual simulation teaching platform to basic theory and clinical teaching of nursing students. Advanced learning methods and realistic clinical cases can not only improve students' interest, but also help students build comprehensive health assessment thinking and adaptability.

Continuing education for medical staff: Most general hospitals regularly or irregularly carry out continuing education lectures or training for some medical staff, but due to the limitation of forms, it is impossible to guarantee that every medical staff can receive enough learning opportunities. Using the virtual health assessment system based on internet plus, all medical staff can visit and learn at any time.

Standardized assessment: At present, medical assessment is still based on examination papers and other single forms, but these assessment methods are not only difficult to achieve the desired results, but also require a lot of human resources. Using the Internet plus virtual simulation health assessment system to assess, the examiners can understand the relevant theoretical knowledge and health assessment ability by means of each learner's assessment report.

Ordinary users: Through experiential exercises on cases developed for ordinary people, ordinary people can understand the assessment process of common diseases (especially acute diseases), which is not only of great significance to save lives at critical moments, but also can improve the healthy quality of life on a daily basis. But also a new mean to popularize medical knowledge.

\subsection{Improve cost-effectiveness}

Traditional medical training equipment is often millions, and students' training opportunities are very limited. By using the virtual health assessment system based on internet plus, geographical restrictions are diluted, and mobile operations such as smart phones and tablet computers are also supported. Learners can learn independently outside the classroom conveniently and flexibly, without being limited by time and place. Training can be carried out locally, which can reduce the cost of course training and travel expenses. Health management departments, education management departments and health science popularization executive departments can assess, teach and publicize medical personnel without increasing workload. The number and frequency of examination and study can be greatly improved, which is conducive to the improvement of the overall technical level of nursing practitioners in China and greatly expands the acquisition of public health knowledge.

\section{Application of Clinical Nursing Health Assessment Decision System Based on Internet+}

The virtual health assessment system based on Internet+ focuses on cultivating the "thinking logic" of nursing students' health assessment, rather than just remembering the medical concepts in books. At the same time, the advanced ability and comprehensive evaluation achievement management mechanism are introduced to stimulate users' learning desire and initiative, and achieve the training goal through the combination of reality and reality. That is to say, every health assessment knowledge and skill of users in this system can be acquired only after online simulation diagnosis and treatment and offline certification are qualified, and managed by the ability management module as a whole, changing the traditional independent discipline system.

The ultimate goal of the project is to accumulate experience through deep integration of industry, university and research, and jointly build industry, university and research resources, and gradually form a virtual health assessment system based on Internet+. Therefore, the quality of clinical care can be better guaranteed, the safety of clinical diagnosis and treatment can be improved, and the health knowledge can be more effectively used in a wider range. 
To seize the opportunity is to take the traditional nursing work as a breakthrough, and explore the development path and method mode to realize digitalization, platformization, online and individuation of nursing work.

\section{Summary}

With the cross-border integration of information technologies such as cloud computing, big data, Internet of Things, mobile Internet, wearable diagnosis and treatment equipment and nursing work, "Internet+ Nursing" has become increasingly closely related. There is an urgent need to build a platform for thinking mode and decision-making of clinical nursing health assessment based on Internet+, so as to serve teaching, clinical nursing practice and the public. However, this is not a simple sum of the two, but the use of information and communication technology and the Internet platform, so that the Internet and traditional industries in-depth integration, to create a new health assessment skills science popularization, health assessment teaching, health assessment clinical application system integration development ecology. How to grasp the opportunity and deal with the challenge is an important topic for nursing workers.

\section{Acknowledgments}

Heilongjiang University Student Innovation and Entrepreneurship Training Project, Item No.: 201812729006.

Qiqihar Science and Technology Research Project, Item No.: 2017GG2381.

\section{References}

[1] Yu Mei; Xu Yan;; Analysis of innovative thinking and development measures under the "Internet + nursing" model [J]; Practical Electronic Journal of Clinical Nursing; 2019-22.

[2] Lu Xiaowen; study the psychology of patients and do a good job of nursing[J]; Chinese National Folk Medicine; 2009-20.

[3] Han Ying; Zhang Pan; Li Xuemei; Liu Junjie; Hao Jing; Zhao Yaning; Li Xiu;; Health assessment teaching design based on "Internet +" thinking[J]; Health Vocational Education; 2019-06.

[4] Guo Daying; PBL+CBL combined teaching method applied to health assessment teaching[J]; Curriculum Education Research; 2017-05.

[5] Wang Zanli; the application of the teaching model of "combining network resources and autonomous learning" in health assessment courses [J]; Curriculum Education Research; 2017-26. 